\title{
GPPS-BJ-2019-0212
}

\section{DEVELOPMENT OF A CORRELATION TO REPRESENT NON-UNIFORM BLADE TIP GAPS WITHIN A COMPRESSOR PERFORMANCE MODEL}

\author{
Tim Sauer \\ Chair for Aero Engines \\ Institute of Aeronautics and Astronautics \\ Technische Universität Berlin \\ Tim.Sauer@ill.tu-berlin.de \\ Berlin, Germany
}

\author{
Dieter Peitsch \\ Chair for Aero Engines \\ Institute of Aeronautics and Astronautics \\ Technische Universität Berlin \\ Dieter.Peitsch@ilr.tu-berlin.de \\ Berlin, Germany
}

\begin{abstract}
In order to cover non-uniform blade tip gaps within a compressor mean line performance program a method is developed that derives a generic correlation that converts a discretized gap of any shape into an equivalent constant clearance applicable for common loss models. This method is demonstrated with the example of the NASA Rotor 37. For this purpose, the efficiency losses of uniform gap geometries are compared with those of non-uniform gaps of the same rotor. The efficiency information is provided by flow simulation studies. Geometric weighting factors have been determined for a correlation function, so that it is now possible to infer uniform from non-uniform gaps. This enables a mean line representation of blades with non-uniform tips.

In the example of the NASA Rotor 37, the standard deviation of the correlation error of the considered member is below the measurement uncertainty for experimental determination of the gap size. Validation cases confirm this result.
\end{abstract}

\section{INTRODUCTION AND OBJECTIVE}

Tip clearance flow effects are well known for their influence on the compressor performance. Therefore, numerous studies have been carried out to understand these effects. For instance in (Smith \& Cumpsty, 1984) as well as in (Wisler, 1985) the impact of the tip flow is quantified while in (Chen, et al., 1991), (Denton, 1993) and (Storer \& Cumpsty, 1993) the tip flow is described analytically. Since these models are limited to a few basic geometric input parameters, complex structures and their associated losses cannot be mapped with common methods. In particular blade tip gaps of an axial compressor are specified as an absolute or even relative scalar value. This may be sufficient for current engines and therefore present loss models such as the approach by Traupel (Traupel, 2001) or Aungier (Aungier, 2003) get by with just this scalar information of the gap geometry. However, non-uniform tip geometries will become more relevant for the future design of compressors, for example to influence secondary flows or as a result of casing treatments or variable geometries. These non-uniform blade tip gaps cannot be addressed by common loss models which leads to an increased uncertainty of the whole performance model.

Therefore, this paper offers a model reduction approach to convert non-uniform gap geometries into a scalar gap information which can be used as an input for current, wellproven tip gap loss models for mean line calculation.

\section{METHODOLOGY}

In order to convert a non-uniform gap into a correlating constant gap geometry, a workflow as shown in Figure 1 is established.

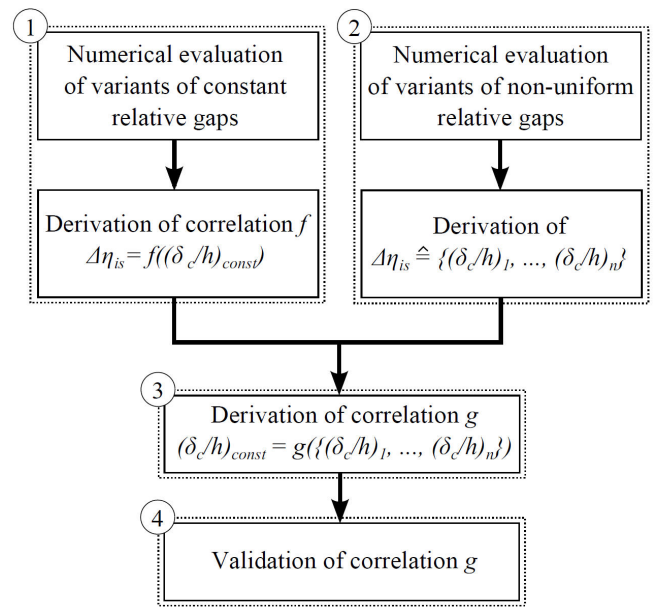

Figure 1 Summarized workflow to derive the correlation between non-uniform gaps and constant gaps 
The workflow can be divided into four major parts:

1) The determination of the correlation $f$ between the efficiency impact $\Delta \eta_{i s}$ and clearances with a constant relative gap $\delta_{c} / h$

2) A study which describes the efficiency impact of non-uniform clearances represented by sets of local relative gaps

3) The derivation of a correlation $g$ that relates nonuniform gaps to representative constants clearances

4) The validation of the correlation $g$

Before these sub processes are described in detail, the numerical approach shall be examined first.

\section{Numerical setup}

The flow simulation study is carried out using the CFD code Fine ${ }^{\mathrm{TM}} /$ Turbo from Numeca with a RANS solver and a Spalart-Allmaras turbulence model (Spalart \& Allmaras, 1994). The subject of the examination is the transonic axial NASA Rotor 37 (Moore \& Reid, 1980) using a systematic variation of the blade tip geometry. The complete stage is not calculated, since effects of the rotor-stator interaction can be mapped via other loss models.

The computational domain as well as the survey planes correspond to the specifications of (Moore \& Reid, 1980). Figure 2 provides an impression of this. Periodic boundary conditions are applied so that only one blade represents the complete rotor.

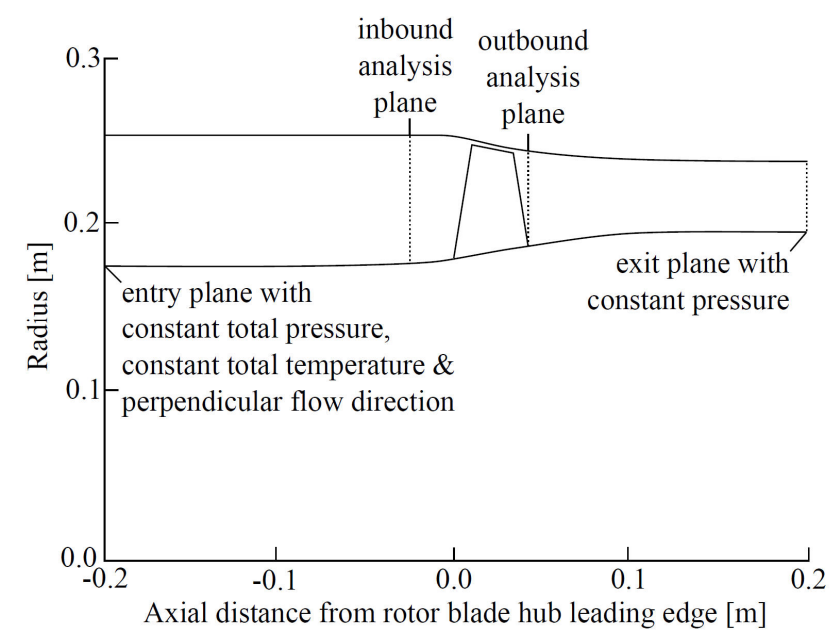

Figure 2 Computational domain of NASA Rotor 37 corresponding to (Moore \& Reid, 1980)

A multiblock $\mathrm{O} 4 \mathrm{H}-\mathrm{Grid}$ is generated using the Autogrid $^{\mathrm{TM}}$ turbomachinery grid code. The grid is kept constant to $90 \%$ span while only the tip geometry of each variant is individually generated. Nevertheless, for each net variant an independency study is carried out, which lead to nets with at least 1.46 million nodes. In order to keep the discretization respectively modelling errors low, all variants with an expansion ratio greater than 2 , a $y^{+}{ }_{\max }$ greater than 5 and a $y^{+}$mean greater than 1.1 are rejected.

The reference case of the NASA Rotor 37 with a nominal gap dimension of $0.4 \mathrm{~mm}$ (Suder \& Celestina, 1996) was calculated with an error of $0.06 \%$ with respect of the pressure ratio $\Pi, 0.11 \%$ with respect of the mass flow $w$ and $0.07 \%$ with respect to the isentropic efficiency $\eta_{i s}$.

\section{Evaluation of constant gap geometries}

In a first step the isentropic efficiency losses of constant tip gaps of different sizes must be determined. In the NASA Rotor 37 example relative gaps with sizes between $\left(\delta_{c} / h\right)_{\text {const }}=0.5 \%$ and $3.75 \%$ are examined. For this purpose, the isentropic efficiencies $\eta_{i s}$ of the constant gap variants are calculated and then deducted from $\eta_{i s}$ of the gapless variant. The resulting $\Delta \eta_{i s}$ represents the losses stemming from the end wall flow field affected by the gap geometry. The calculated reference correlation, which defines the efficiency drop $\Delta \eta_{i s}$ as a function of the constant relative gap $\left(\delta_{c} / h\right)_{\text {const }}$ is shown in Figure 3. It can be seen that the resulting slope of the calculated correlation corresponds to those presented in (Jefferson \& Turner, 1958) and (Mahler, 1972). While the regression line has an increase of $-1.946 \%$ at an $R^{2}$ of 0.9982 , the tangent of Mahler has an increase of $-1.971 \%$ and that of Jefferson and Turner has an increase of $-1.908 \%$. The correlation is therefore considered as validated. Non-linear effects, which occur with very small relative gaps, are excluded in the sense of correlatability. The domain of the correlation is therefore limited to $\left(\delta_{c} / h\right)_{\text {const }}$ greater than 0.5 .

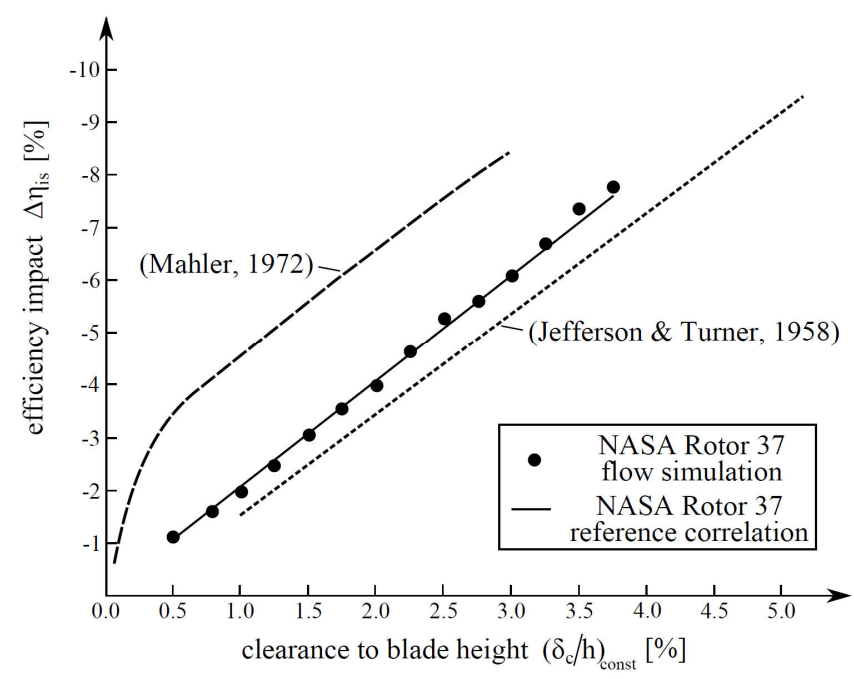

Figure 3 Reference correlation of NASA Rotor 37: efficiency impact $\Delta \eta_{\text {is }}$ over constant relative tip gap $\left(\delta_{c} / h\right)_{\text {const }}$

According to the collected results in (Ludwig \& Bill, 1980), an efficiency loss of $1.95 \%$ is added for each $1 \%$ additional relative gap. The correlation $f$ therefore results in Equation 1, valid for NASA Rotor 37.

$$
\Delta \eta_{\text {is }}=f\left(\left(\frac{\delta_{c}}{h}\right)_{\text {const }}\right)=-0.0195 \cdot\left(\frac{\delta_{c}}{h}\right)_{\text {const }}
$$


It can be seen that $f$ is a bijective function. This means that $\Delta \eta_{i s}$ can also be used to draw conclusions about the existing gap.

\section{Evaluation of non-uniform gap geometries}

In the next step, 43 randomly created tip geometries for the NASA Rotor 37 are generated. The parameter space used for this purpose consists of seven support points distributed equally over the chord $s$. Each support point can vary between $0.5 \%$ and $6.0 \%$ of $\delta_{c} / h$. The resulting tip results from a cubic spline interpolation of these points. Figure 4 shows this approach graphically.

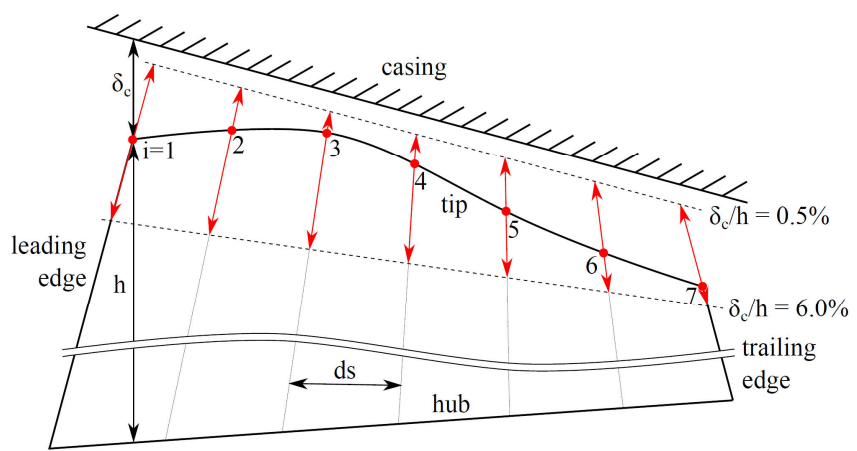

Figure 4 Parameter space for generation of non-uniform gaps

Analogous to the calculation of the efficiency effects of the constant gap geometries, the determined $\eta_{i s}$ are deducted from $\eta_{i s}$ of the gapless variant. So each non-uniform gap variant results into an $\Delta \eta_{i s, j}$ representing the end wall losses based on the respective gap geometry, which is specified in the particular set $M_{j}=\left\{\left(\delta_{c} / h\right)_{1}, \ldots,\left(\delta_{d} / h\right)_{7}\right\}_{j}$. This $\Delta \eta_{i s, j}$ enables the connection of a non-uniform gap geometry with a uniform one.

\section{Derivation of correlation $g$}

In order to convert a non-uniform gap geometry into a uniform one, a correlation $g$ must be found, which translates the local relative gaps into an integral relative gap without the use of additional flow simulations. For this purpose, the utilized sets $M_{j}$ with their corresponding $\Delta \eta_{i s, j}$ are assigned to their respective constant relative gap using the correlation $f$. This makes it possible to set up a regression function with an order equal to the number of support points, i.e. 7. This regression function, which at the end will yield the desired correlation $g$, must be capable to satisfy the hypothetical test cases shown in Figure 5. These test cases are introduced subsequently.

\section{1) Generic non-uniform tip}

The first test case describes the primary goal of the correlation: the representation of a generic non-uniform tip geometry. Here it becomes apparent that the equivalent constant gap belonging to a non-uniform gap geometry commonly does not correspond to the simple mean value of the local relative gaps. Therefore, the correlation $g$ must be capable to reproduce the integral relative gap by means of weighting factors of the individual local relative gaps. This implies that the impact of the particular local gap varies along the chord. The reason for this is the varying degrees of impact of the respective local gaps on the development of losses.

A linear weighting function is preferred, since this corresponds to the character of the reference correlation $f$. Furthermore, the parameter space of the correlation $g$ is not unnecessarily distended.

\section{2) Generic uniform tip}

The second test case describes the conventional design of a uniform blade tip. This case may seem trivial, since the resulting equivalent gap is exactly as large as the local gaps, but it is an essential specification for the mathematical formulation of correlation $g$. In concrete terms, this means that the order of magnitude of the resulting equivalent gap must not be derived from an absolute origin, but must be inherently derived from the local gaps. Otherwise an approach using position specific weighting factors would lead to an averaging of a non-uniform gap distribution. The resulting equivalent gap would deviate from the given uniform gap.

\section{3) Tip with local deviation}

The last test case describes a uniform tip geometry with a local deviation. This leads to another important specification of the mathematical formulation of $g$. Since this case represents the behaviour towards outliers, it becomes obvious that the inherent determination of the magnitude of the resulting equivalent gap must not depend on the arithmetic mean of the local gaps. Otherwise, weighting factors would be applied to the unaffected local gaps, allowing the resulting equivalent gap to be smaller than the original gap. Therefore, the median of the local relative gaps $\left(\widehat{\delta_{c} / h}\right)_{M}$ is used instead of the arithmetic mean, since this is much more robust against outliers.

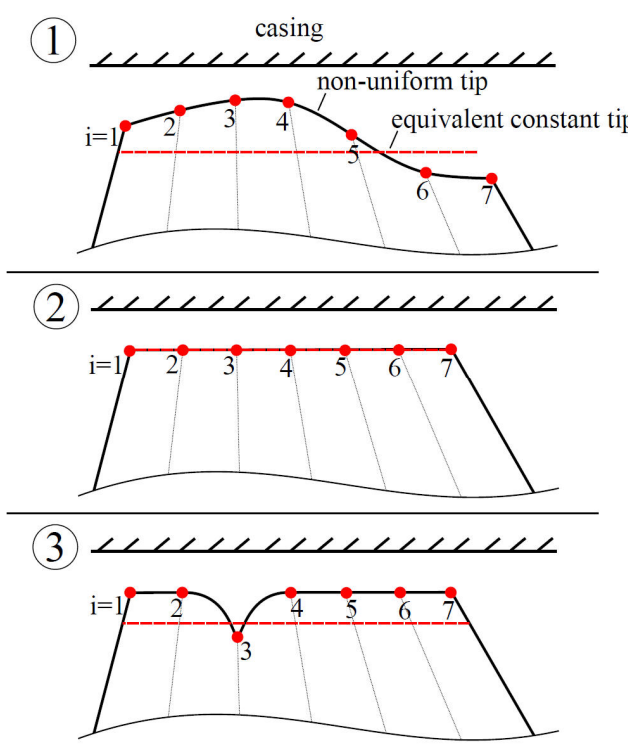

Figure 5 Test cases for correlation $g$ : 1) generic nonuniform tip, 2) generic uniform tip, 3) tip with local deviation 
Based on these test cases, the correlation $g$ is defined as follows:

$$
\begin{aligned}
\left(\frac{\delta_{c}}{h}\right)_{\text {corr }} & =g(M)=g\left(\left\{\left(\frac{\delta_{c}}{h}\right)_{1}, \ldots,\left(\frac{\delta_{c}}{h}\right)_{7}\right\}\right) \\
& =\widetilde{\left(\frac{\delta_{c}}{h}\right)_{M}}+\frac{1}{7} \sum_{i=1}^{7} \begin{cases}\alpha_{i} \cdot\left(\left(\frac{\delta_{c}}{h}\right)_{i}-\overline{\left(\frac{\delta_{c}}{h}\right)_{M}}\right),\left(\frac{\delta_{c}}{h}\right)_{i} \geq \overline{\left(\frac{\delta_{c}}{h}\right)_{M}} \\
\beta_{i} \cdot\left(\left(\frac{\delta_{c}}{h}\right)_{i}-\overline{\left(\frac{\delta_{c}}{h}\right)_{M}}\right),\left(\frac{\delta_{c}}{h}\right)_{i}<\overline{\left(\frac{\delta_{c}}{h}\right)_{M}}\end{cases}
\end{aligned}
$$

The accuracy of the correlation $g$ is increased by a case distinction of the weighting factors. The factor $\alpha_{i}$ is used if the local relative gap is greater than or equal to the median $\left(\widetilde{\delta_{c} / h}\right)_{M}$. Otherwise the factor $\beta_{i}$ is used. The factors are determined via a root mean square approach, which is represented by Equation (3). The rms function is minimized using a genetic algorithm with a subsequent interior point algorithm. The initial values are varied randomly until a solution is smaller than the mean value of the previous solutions reduced by the triple standard deviation of the previous solutions. This solution represents the final one.

$$
\begin{gathered}
r m s=\sqrt{\frac{1}{n} \sum_{j=1}^{n}\left(\left(\frac{\delta_{c}}{h}\right)_{c o r r, j}-\left(\frac{\delta_{c}}{h}\right)_{c o n s t, j}\right)^{2}} \\
\text { with }\left(\frac{\delta_{c}}{h}\right)_{c o r r, j}=g\left(M_{j}\right) \text { and }\left(\frac{\delta_{c}}{h}\right)_{c o n s t, j}=f^{-1}\left(\Delta \eta_{i s, j}\right)
\end{gathered}
$$

The number of members available to calibrate the correlation is represented by $n$. In order to characterise the behaviour of the correlation $g$ with respect to overfitting, the split between calibration cases and validation cases is varied. To decrease the impact of potential outliers during this survey, each split is examined with five different combinations of members. Figure 6 illustrates the resulting average $r m s$ in dependency of the split ratio after minimization.

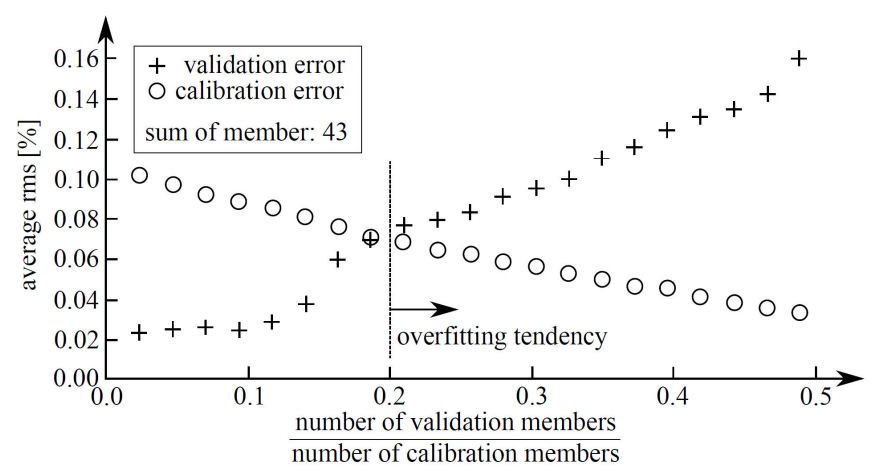

Figure 6 Correlation error of $g$ in dependency of the split ratio between validation to calibration cases

As can be seen, the correlation error decreases with fewer calibration cases. Simultaneously, the error of the validation cases increases. This indicates an overfitting of $g$. The point of intersection between validation and calibration error is seen to be the threshold. That means that if less than 35 members are used for calibration, the correlation $g$ tends towards single case solutions. In order to be able to provide enough validation cases, $n$ is specified to 35 .

\section{RESULTS AND DISCUSSION}

The weighting factors of the correlation $g$ for the NASA Rotor 37 are summarized in Table 1.

Table 1 Weighting factors of correlation $g$ for NASA Rotor 37

\begin{tabular}{c|c|c}
$i$ & $\alpha_{i}$ & $\beta_{i}$ \\
\hline 1 & 0.65921 & 0.94514 \\
2 & 0.11167 & 2.32535 \\
3 & 0.38729 & 3.09600 \\
4 & 0.00002 & 1.64385 \\
5 & 0.00001 & 3.07088 \\
6 & 0.41520 & 2.15015 \\
7 & 0.06389 & 1.80824
\end{tabular}

Figure 7 gives a graphical impression of the individual weighting factors for the NASA Rotor 37. It illustrates the impact of the weighting factors on the deviation $k$ between the particular local relative gaps and their joint median. As can be seen, there is a strong dependence for all local gaps except the one at the leading edge (i.e. $i=1$ ) regarding their relative position to the median. The gaps that are smaller than the median are much more strongly corrected than those that are larger. This means that the correlated integral gap is much closer to the local gaps, which are particularly small. Therefore, it seems that the size of the smaller local gaps has a greater impact on the efficiency loss than the size of the greater local gaps.

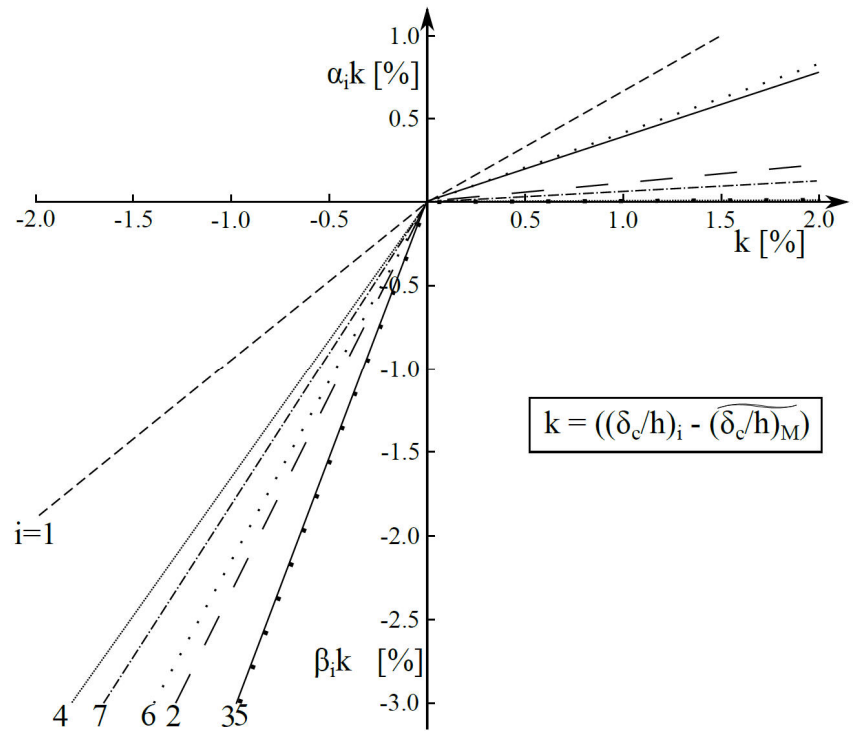

Figure 7 Impact of weighting factors of correlation $g$ for NASA Rotor 37

As mentioned above, the relative gap at the leading edge must be treated separately. It can be seen here that large clearances have an above-average impact on the total loss. In addition, small clearances have a much lower impact on the correlating integral gap. Both imply that the blade overflow 
here reacts much more sensitively to a gap enlargement than downstream.

Figure 8 shows the result of correlation $g$ applied to the members. In addition, the interval of the local relative gaps of the respective members is presented. Once again it can be seen that the correlated integral gap is much closer to the local gaps, which are particularly small. The dominance of the smaller local gaps in terms of efficiency loss is thus confirmed.

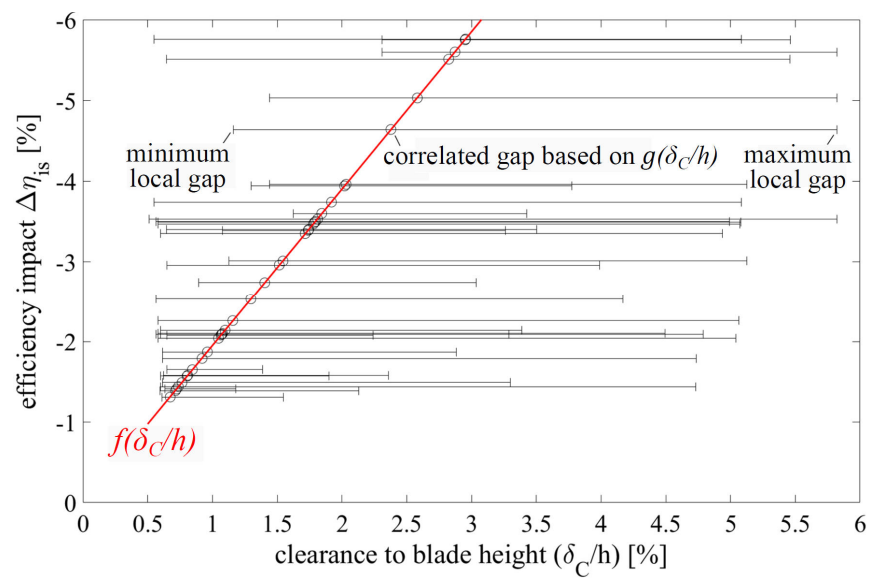

Figure 8 Outcome of correlation $g$ according to the respective interval of the local gaps and comparison with reference correlation $f$

The accuracy of the correlation $g$ becomes evident by the comparison with the reference correlation $f$. Figure 9 shows the efficiency error between the correlation $f$ and the results of correlation $g$ for all calibration members. Additionally, the errors of the 8 validation members are depicted. They show a similar order of magnitude, confirming that overfitting did not take place.

The simple standard deviation of the error between the calculated efficiency of the non-uniform gap and the efficiency resulting from the reference correlation of the corresponding constant gap equivalent is smaller than the experimental measurement uncertainty for the radial gap determination of NASA Rotor 37 (Suder \& Celestina, 1996). Thus, the efficiency error made by the correlation $g$ is acceptably small.

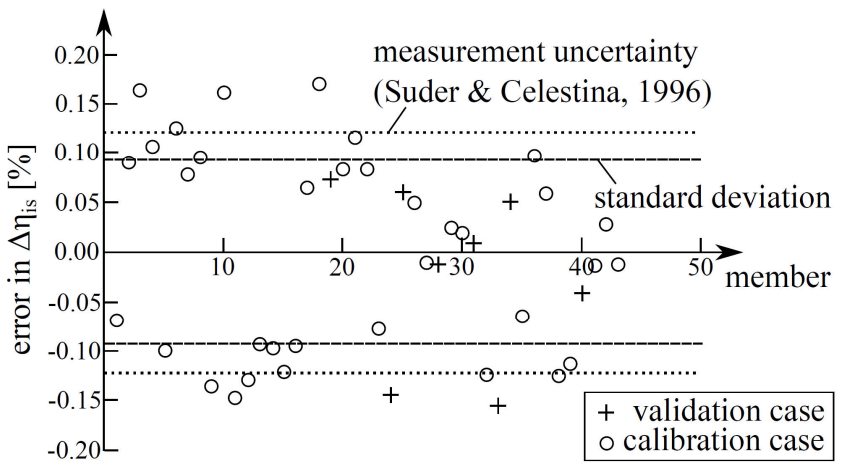

Figure 9 Efficiency error between correlation $f$ and outcome of correlation $g$ for NASA Rotor 37

\section{CONCLUSIONS}

In this paper an approach is presented, which yields a correlation that converts a non-uniform compressor blade tip clearance into an equivalent constant gap, suitable for common loss models of mean-line performance programs. The presented method is demonstrated using a tip gap variation of the NASA Rotor 37. The following conclusions are drawn for this case:

- The efficiency loss depends linearly on the integral relative gap $\delta_{c} / h$, assuming that the relative gap is greater than $0.5 \%$. Below this threshold, a superlinear relationship exists.

- Local gaps smaller than the median of the discretized gap are weighted with a greater factor than large local gaps. The smaller local gaps of the discretized gap thus dominate the order of magnitude of the equivalent constant gap.

- $\quad$ The blade overflow at the leading edge reacts much more sensitively to a gap enlargement than downstream. This means that a large clearance at the leading edge of the blade has an above-average impact on the efficiency loss.

It is recommended that the correction factors $\alpha$ and $\beta$ of the correlation are re-determined for other profile families. For this purpose, the presented approach can be used:

1) Definition of a reference correlation that reflects the efficiency deficit of constant gaps.

2) Determination of the efficiency loss of variants with non-uniform gaps, discretized as described above.

3 ) Determination of the correction factors of the correlation frame specified in Equation (2) using the efficiency loss parity within a rms approach. At least 35 calibration cases are recommended to avoid overfitting.

4) Validation using retained data.

The presented correlation is defined in such a way that it covers any non-uniform blade tip geometry. This will become relevant for future compressors with innovative blade tip design or even with variable pitch rotors.

\section{NOMENCLATURE}

() median of ( )

$f$ reference correlation

$g \quad$ transfer correlation

$h \quad$ blade height

$i \quad$ index of local relative gap

$j \quad$ index of member item

$k$ deviation

$M \quad$ set of local relative gaps

$n \quad$ number of member

$R^{2} \quad$ coefficient of determination

$r m s$ root mean square

$s \quad$ chord

w mass flow 
$y^{+} \quad$ dimensionless wall distance

$\alpha \quad$ weighting factor

$\beta \quad$ weighting factor

$\Delta \quad$ differential

$\delta_{c} \quad$ absolute radial blade tip clearance

$\eta \quad$ efficiency

$\Pi \quad$ pressure ratio

\section{Subscripts}

$\begin{array}{ll}\text { const } & \text { constant } \\ i & \text { index of local relative gap } \\ i s & \text { isentropic } \\ j & \text { index of member item } \\ M & \text { belonging to } M \\ \text { max } & \text { maximum } \\ \text { mean } & \text { arithmetic mean }\end{array}$

\section{ACKNOWLEDGEMENT}

The authors acknowledge David van Zyl for his support in this research.

\section{REFERENCES}

Aungier, R. H., 2003. Axial-Flow compressors : a strategy for aerodynamic design and analysis. New York: The American Society of Mechanical Engineers.

Chen, G. T., Greitzer, E. M., Tan, C. S. \& Marble, F. E., 1991. Similarity Analysis of Compressor Tip Clearance Flow Structure. ASME Journal of Turbomachinery, Issue 113, pp. 260-271.

Denton, J. D., 1993. Loss Mechanisms in Turbomachines. ASME Journal of Turbomachinery, Issue 115, pp. 621-656.

Jefferson, J. L. \& Turner, R. C., 1958. Some Shrouding and Tip Clearance Effects in Axial Flow Compressors. Int. Shipbuilding Progress, 5, Issue 42, pp. 78-101.

Ludwig, L. P. \& Bill, R. C., 1980. Gas Path Sealing in Turbine Engines. ASLE TRANSACTIONS, Issue 23, pp. 1-22.

Mahler, F. H., 1972. Advanced Seal Technology, East Hartford, Conn.: PWA-4372, Pratt \& Whitney Aircraft.

Moore, R. D. \& Reid, L., 1980. Performance of SingleStage Axial-Flow Transonic Compressor With Rotor and Stator Aspect Ratios of 1.19 and 1.26, Respectively, and With Design Pressure Ratio of 2.05, Cleveland: NASA Technical Paper 1659.

Smith, G. D. J. \& Cumpsty, N. A., 1984. Flow Phenomena in Compressor Casing Treatment. ASME Journal of Engineering for Gas Turbines and Power, Issue 106, pp. pp. 532-541.

Spalart, P. R. \& Allmaras, S. R., 1994. A One-Equation Turbolence Model for Aerodynamic Flows. Recherche Aerospatiale, Issue 1, pp. 5-21.

Storer, J. A. \& Cumpsty, N. A., 1993. An Approximate Analysis and Prediction Method for Tip Clearance Loss in Axial Compressors. ASME Journal of Turbomachinery, Issue 116, pp. 648-656.

Suder, K. L. \& Celestina, M. L., 1996. Experimental and Computational Investigation of the Tip Clearance Flow in a Transonic Axial Compressor Rotor. ASME Journal of Turbomachinery, Issue 118, pp. 218-229.
Traupel, W., 2001. Thermische Turbomaschinen Bd. 1. Thermodynamisch-strömungsmechanische Berechnungen. 4. ed. Berlin; Heidelberg: Springer.

Wisler, D. C., 1985. Loss Reduction in Axial Flow Compressors Through Low-Speed Model Testing. ASME Journal of Turbomachinery, Issue 107, pp. 354-363.

\section{APPENDIX - UTILISED MEMBER}

The following 43 members were used to establish the correlation $g$ of the NASA Rotor 37. The data is presented in percentage terms.

\begin{tabular}{|c|c|c|c|c|c|c|c|c|}
\hline & $\left(\frac{\delta_{c}}{h}\right)_{1}$ & $\left(\frac{\delta_{c}}{h}\right)_{2}$ & $\left(\frac{\delta_{c}}{h}\right)_{3}$ & $\left(\frac{\delta_{c}}{h}\right)_{4}$ & $\left(\frac{\delta_{c}}{h}\right)_{5}$ & $\left(\frac{\delta_{c}}{h}\right)_{6}$ & $\left(\frac{\delta_{c}}{h}\right)_{7}$ & $\left(\frac{\delta_{c}}{h}\right)_{c}$ \\
\hline & 0.646 & 2.310 & 3.425 & 4.098 & & & & \\
\hline & & & & & & & & \\
\hline & & & & & & & & \\
\hline & 468 & 199 & 340 & & & & 860 & \\
\hline & 700 & 423 & 580 & & & & & \\
\hline & & & & & & & & \\
\hline & 623 & & & & & & & \\
\hline & 821 & & & & & & & \\
\hline & 551 & & & & & & & \\
\hline & & & & & & & & \\
\hline & 945 & 54 & & 714 & & & & \\
\hline & & & & & & & & \\
\hline & & & & & & & & \\
\hline 4 & 27 & & & & & & & \\
\hline & & & & & & & & \\
\hline & & & & & & & & \\
\hline 1 & & & & & & & & \\
\hline & & & & & & & & \\
\hline 9 & & & & & & & & \\
\hline 20 & & & & & & & & \\
\hline 1 & & & & & & & & \\
\hline & 93 & & & & & & & \\
\hline 3 & 5 & & & & & & & \\
\hline 64 & & & & & & & & \\
\hline 5 & & & & & & & & \\
\hline 5 & & & & & & & & \\
\hline 7 & & & & & & & & \\
\hline 8 & & & & & & & & \\
\hline & & & & & & & & \\
\hline 30 & & & & & & & & \\
\hline 1 & & & & & & & & \\
\hline & & & & & & & & \\
\hline 3 & & & & & & & & \\
\hline$T$ & & & & & & & & \\
\hline & & & & & & & & \\
\hline 36 & & & & & & & & \\
\hline 37 & 5 & & & & & & & \\
\hline , & & & & & & & & \\
\hline 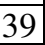 & & & & & & & & \\
\hline 40 & & & & & & & & \\
\hline 41 & & & & & & & & \\
\hline & & & & & & & & \\
\hline & & 704 & & 5 & & & 5 & \\
\hline
\end{tabular}

